\title{
Recherches en éducation
}

29 | 2017

La modélisation des savoirs dans les analyses didactiques des situations d'enseignement et apprentissage

\section{Modélisation des problématisations historiques en classe et chez les historiens}

Modelization of historical problematizations in the classroom and among

historians

Sylvain Doussot

\section{OpenEdition}

Journals

Édition électronique

URL : http://journals.openedition.org/ree/2917

DOI : $10.4000 /$ ree. 2917

ISSN : 1954-3077

Éditeur

Université de Nantes

Référence électronique

Sylvain Doussot, "Modélisation des problématisations historiques en classe et chez les historiens », Recherches en éducation [En ligne], 29 | 2017, mis en ligne le 01 juin 2017, consulté le 08 novembre 2020. URL : http://journals.openedition.org/ree/2917 ; DOI : https://doi.org/10.4000/ree.2917

\section{$(1) \Theta$}

Recherches en éducation est mise à disposition selon les termes de la Licence Creative Commons Attribution - Pas d'Utilisation Commerciale - Pas de Modification 4.0 International. 


\title{
Modélisation des problématisations historiques en classe et chez les historiens
}

\author{
Sylvain Doussot ${ }^{1}$
}

\begin{abstract}
Résumé
L'opération qui consiste à produire des modèles de la réalité constitue une activité centrale dans la plupart des démarches scientifiques. En didactique la modélisation peut jouer un rôle médiateur dans la comparaison entre une discipline scolaire et sa référence scientifique. C'est dans ce sens que l'on propose d'aborder la modélisation des savoirs élaborée par les travaux inscrits dans le cadre théorique de la problématisation. Une des spécificités de la modélisation des problématisations consiste à tenir ensemble les textes de savoir et les pratiques de mise en texte de ces savoirs. Cependant, le risque inhérent à toute modélisation réside dans la tendance à ignorer l'historicité des activités que l'on vise à modéliser. Une telle dérive objectiviste laisserait de côté la dimension d'incertitude qui caractérise toute pratique. Cet article vise à élucider les conditions de la prise en charge de ce risque par le biais de l'étude d'une séquence élaborée avec l'enseignante sur la Révolution en classe de fin d'enseignement primaire. L'étude aboutit à envisager l'étude de cas comme modélisation de la problématisation historique, dont on discerne le caractère heuristique à deux niveaux de temporalités différents.
\end{abstract}

L'opération qui consiste à produire des modèles de la réalité constitue une activité centrale dans la plupart des démarches scientifiques, elle intéresse donc potentiellement la recherche en didactiques disciplinaires du point de vue des modélisations des savoirs et des apprentissages. Qu'elle soit descriptive ou interprétative, la modélisation est un moyen d'appréhender l'enseignement-apprentissage et peut en particulier jouer un rôle médiateur dans la comparaison entre une discipline scolaire et sa référence scientifique. C'est dans ce sens que l'on propose d'aborder la modélisation des savoirs élaborée par les travaux inscrits dans le cadre théorique de la problématisation (Fabre, 2009 ; Orange, 2005). Nous l'abordons dans cet article pour le cas de la didactique de l'histoire en considérant que la modélisation constitue une médiation entre les problématisations historiques scientifiques (celles des historiens) et les problématisations historiques scolaires, permettant d'éviter le double écueil d'une analogie non contrôlée et d'une séparation insurmontable entre les deux mondes en jeu (scolaire et scientifique). Une des spécificités de la modélisation des problématisations consiste à tenir ensemble les textes de savoir et les pratiques de mise en texte de ces savoirs, en ce sens ce cadre théorique envisage les savoirs comme essentiellement liés aux activités qui les rendent possibles.

Cependant, en didactique comme dans d'autres domaines scientifiques, le risque inhérent à toute modélisation réside dans la tendance à ignorer l'historicité des activités que l'on vise à modéliser en faisant de celles-ci des objets d'observation et d'analyse. Une telle dérive objectiviste laisserait de côté la dimension d'incertitude qui caractérise toute activité pratique en tant qu'elle est nécessairement ancrée dans l'histoire, ce que les sciences sociales et historiques savent bien. C'est la prise en charge de ce risque que cherche à élucider ce texte pour le cas des modélisations de savoir en terme de problématisation. II s'y engage par le biais de l'étude d'une séquence sur l'enseignement de la Révolution en classe de fin d'enseignement primaire.

\footnotetext{
${ }^{1}$ Maître de conférences, HDR, en didactique de l'histoire, Centre de recherche en éducation de Nantes (CREN), ESPE, université de Nantes.
} 


\section{Savoirs sur le passé et savoirs historiques problématisés}

Pour étudier les conditions d'accès des élèves à des savoirs historiques sur le passé, il convient de caractériser la différence entre des savoirs de sens commun et des savoirs scientifiques. Dans le cadre théorique de la problématisation, la différence entre les deux réside dans le fait que les savoirs scientifiques rendent raison de la pertinence de l'explication proposée par rapport aux autres explications possibles, alors que le sens commun se contente de la cohérence non questionnée d'une explication convaincante. En histoire, on peut formuler cette caractéristique en répondant par la négative à la question : "Peut-on comprendre pourquoi les choses se sont passées comme elles l'ont fait, sans se demander si elles auraient pu se passer autrement ? » (Prost, 1996). L'enquête historique peut, sous cet angle épistémologique et didactique, être modélisée en premier lieu comme l'exploration et la délimitation du champ des explications possibles : "Derrière la question "comment en est-on arrivé là ?" se cache la question de savoir comment il était même possible qu'on en arrivât là » (Koselleck, 1997). Cette perspective est une manière de rendre compte de l'incertitude des situations passées du point de vue des acteurs et de se garder de la tentation téléologique propre à la position de l'historien qui vient après et qui connaît la suite de l'histoire ${ }^{2}$. De ce point de vue, les possibles de l'explication historique ont directement à voir avec les possibles des acteurs de l'événement ou du phénomène étudié : reconstituer le champ des possibles c'est décrire l'incertitude vécue en relation avec les déterminants conscients ou inconscients des acteurs. Cette reconstruction est pensée de manière spécifique, dans le cadre théorique de la problématisation, comme une « exploration et une cartographie » (Orange, 2005) du champ des possibles qui conduit à définir des impossibilités, des probabilités et des nécessités, ce qui caractérise le savoir comme apodictique $^{3}$. En ce sens, ce travail d'enquête est productif parce qu'il ne se contente pas, par exemple, d'évoquer des possibles non advenus ${ }^{4}$, mais repose sur une activité systématique de délimitation et de reconstruction de chemins possibles pour parvenir à dire pourquoi les choses ne se sont pas passées autrement.

Par contraste, dans le sens commun (des élèves, mais pas seulement) la mise en jeu de traces du passé conduit avant tout à produire des savoirs assertoriques qui utilisent ces traces pour argumenter une explication sans la confronter explicitement et systématiquement à d'autres possibles. Dans le travail scolaire ordinaire ${ }^{5}$, les traces sont appréhendées comme des données qui alimentent des raisonnements fondés sur des modèles de comportements de l'expérience sociale partagée, et dont on n'interroge pas la pertinence explicative. Par exemple, comme nous le verrons dans le cas développé dans l'exemple analysé plus loin, le modèle « misère + injustice = révolte » peut s'appuyer sur des documents tels que les cahiers de doléances pour être appliqué à la période révolutionnaire aussi bien qu'à la situation de la population tunisienne des années 2000. De telles explications se suffisent généralement à elles-mêmes et constituent parlà des obstacles épistémologiques: il est inutile d'explorer d'autres possibles puisque l'explication fournie est convaincante et partagée. L'effort proprement scientifique réside précisément dans le dépassement de cette première satisfaction, par la recherche de données ou de modèles qui entrent en tension entre eux, tension qui conduit à reconstruire le problème. Pour les historiens il ne s'agit par exemple plus seulement de répondre à la question qu'est-ce qui a provoqué la Révolution ? mais de savoir ce qui a changé en 1789 pour que la misère et l'injustice conduisent à ce moment-là à la révolte ${ }^{6}$. On voit en outre, sur cet exemple, que le type de savoir issu d'un tel travail historique de problématisation ne repose pas sur une explication

\footnotetext{
${ }^{2}$ Selon Revel (2001, p. 109) l'événement en histoire « sert aujourd'hui à réfléchir sur l'entrelacement des durées historiques, sur l'expérience des acteurs et il peut permettre de réfléchir sur l'incertitude qui caractérise cette expérience et que les historiens tendent, par position (parce qu'ils viennent après), à ignorer ».

${ }^{3}$ Par opposition au savoir assertorique, dont la vérité n'est pas démontrée.

${ }^{4}$ Comme le suggèrent les références plus ou moins anciennes à l'histoire contre-factuelle (évoquée dans la synthèse de Prost (1996), récemment remises au goût du jour (Deluermoz \& Singaravélou, 2016) et présente dans la fiction).

${ }^{5}$ Notamment décrit par toute une série d'études fondatrices de la didactique de l'histoire (ou de l'histoire-géographie) en France ; voir par exemple (Audigier, 1995 ; Lautier, 1997 ; Tutiaux-Guillon, 2009).

${ }^{6}$ On peut également citer la problématique exposée par Chartier (2000) qui montre comment l'historiographie passe de la question de savoir comment les philosophes des Lumières ont pu influencer les Révolutionnaires, à celle qui se demande comment on parlerait aujourd'hui des Lumières si la Révolution n'avait pas eu lieu.
} 
simplement chronologique et causale mais sur un dépassement de cette linéarité explicative en articulant différentes temporalités (à la temporalité longue du modèle initial se greffe la temporalité courte de la période pré-révolutionnaire).

Dans notre perspective didactique, nous nous intéressons aux conditions du passage du processus de sens commun au processus scientifique de mise en tension des données et des modèles. Pour y parvenir, le savoir sur le passé est modélisé de manière à rendre compte des données et modèles, des relations qui les lient, et des processus d'exploration et de cartographie des explications possibles.

\section{Un exemple de modélisation de problématisation historique scolaire}

Une séquence de trois séances est construite avec une enseignante pour sa classe de CM2 (dernière année du primaire) sur le début de la Révolution ${ }^{7}$. Elle constitue une séquence «forcée » au sens de Orange (2010), c'est-à-dire que la négociation entre enseignante et chercheurs se fait sur la base d'une répartition des responsabilités: elle tient les enjeux pédagogiques tandis que les chercheurs tiennent les enjeux de recherche, ceux-ci visant à produire des phénomènes didactiques intéressants mais dans le contexte "écologique » d'une vraie classe. Les deux premières séances sont organisées autour du visionnage d'un film de fiction intitulé 1788 qui raconte la vie d'un village de Touraine entre l'été 1788 et l'été $1789^{8}$. Le film est vu en deux parties et, à chaque séance, les élèves travaillent sur deux tableaux qu'ils doivent remplir (par deux) pour dire ce qu'ils ont compris de la situation ("difficultés » et « avantages ») du comte et de celle des paysans. En voici un exemple ci-après (Figure 1).

Ce travail conduit les élèves à sélectionner dans le film les éléments qu'ils comprennent et qui correspondent à l'explication qu'ils parviennent à fournir de la révolte des paysans. Cette explication repose en particulier sur le modèle de comportement suivant : la misère et l'injustice que subissent les paysans les poussent à se révolter.

Dans une troisième séance, l'enseignante et les chercheurs visent à mettre en discussion cette explication dominante selon les enseignements issus de recherches antérieures, qui font de la problématisation historique un processus de mise en crise explicite des représentations des élèves, considérées dans leur dimension explicative (non pas les connaissances qu'ils en auraient, mais les explications qu'ils sont capables d'en donner). Pour y parvenir, ils organisent un dispositif qui est susceptible de soutenir le problème qui va être posé dans cette séance : si la misère et l'injustice expliquent la révolte des paysans, comment se fait-il qu'ils attendent 1789 pour se révolter alors que leur situation n'a pas fondamentalement changé ?

Le dispositif de cette troisième séance se structure sur la reprise en vis-à-vis de deux scènes du film: une délégation de quelques paysans se rend auprès du comte pour demander son indulgence, qu'il rejette avec dédain, en 1788 ; l'ensemble du village qui pénètre de force dans le château du comte avec des fourches et des faux, en 1789 (ce qui représente l'épisode historique connu sous le nom de Grande Peur). La question posée, et préparée d'abord individuellement puis par deux sur un support papier, est la suivante: comment expliquer que les paysans se révoltent en 1789 mais pas en 1788 alors qu'ils sont déjà dans la misère et l'injustice ? Un débat d'une demi-heure est animé par l'enseignante sur la base des préparations écrites.

\footnotetext{
${ }_{8}^{7}$ Cette séquence a fait l'objet d'une présentation et d'une analyse ailleurs (Doussot, 2015).

${ }^{8}$ Le film en question met en scène quelques personnages archétypaux (un comte, un bourgeois, un curé, des paysans de statuts et de conditions variés) dont les actions sont reliées aux événements nationaux (sècheresse, crise financière, États généraux, rédaction des cahiers de doléances, envoi de délégués). II a été réalisé en étroite collaboration avec des historiens (un livre a été édité lors de la sortie du film (Failevic \& La Rochefoucauld (de), 1978) par les auteurs et préfacé par A. Soboul, alors le plus reconnu des spécialistes de la période révolutionnaire) et présente un caractère réaliste (et même « didactique » dans le sens commun du terme : qui vise à instruire). Dans cette séquence, le film, bien qu'il soit une fiction, est envisagé comme une source dans le travail historique de la classe: nous ne discutons pas ici des questions que pose ce type de document pour nous contenter d'y voir la source de données et de modèles pris en charge par la classe.
} 
Ce débat, une fois transcrit, peut faire l'objet d'une modélisation en terme d'espace de contraintes (sur la base des catégories précédemment évoquées : données, modèles, possibles, nécessités...) comme dans le tableau 1 ci-après qui en constitue une représentation graphique.

Figure 1 - Travail initial sur le film (le trait horizontal marque la séparation entre les deux séances)

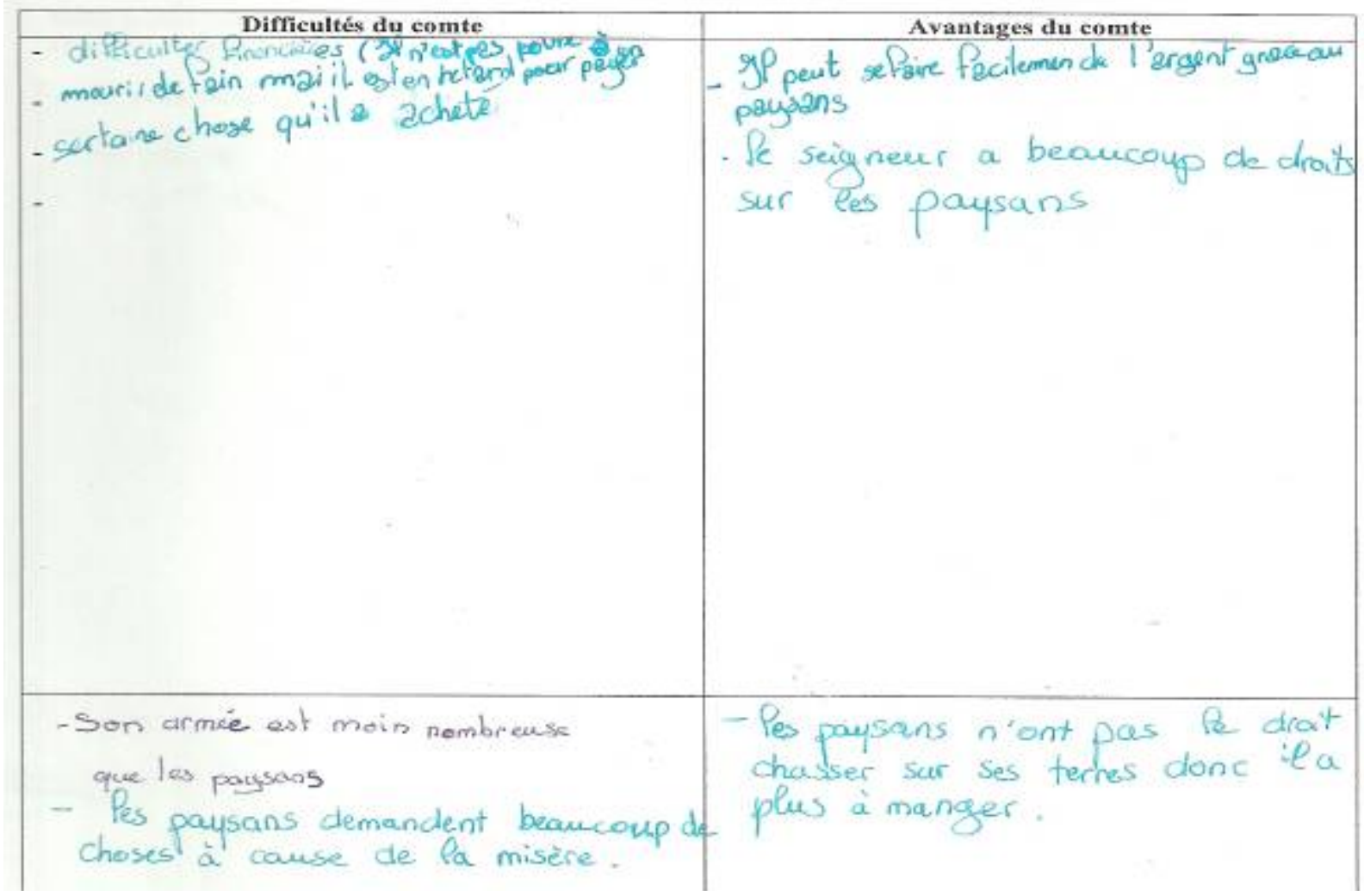

\section{Une modélisation au service de l'analyse du chercheur}

Cette modélisation, dont une représentation possible est proposée ici sous forme de tableau, permet au chercheur d'engager une interprétation de ce qui se passe dans la classe du point de vue du processus de problématisation historique, en référence à la problématisation scientifique.

Dans cette situation de classe, les données peuvent provenir aussi bien du film que des savoirs antérieurs des élèves ou d'éléments fournis par l'enseignante, elles s'inscrivent dans le registre empirique par contraste avec le registre des idées explicatives. Celui-ci constitue le registre des modèles, qui rend compte des modèles de comportements humains qu'utilisent les élèves (sans qu'ils les pensent comme tels) pour expliquer le monde social. II rend également compte des nécessités et des impossibilités sur ces modèles, qui sont construites au cours de la discussion. Le registre explicatif, enfin, constitue le monde mental qui donne du sens aux modèles et qui permet de les manipuler (Orange, 1997) : le registre explicatif chrono-causal associe ainsi des faits successifs selon le raisonnement post hoc ergo propter hoc dans lequel l'antériorité a valeur de causalité. 
Tableau 1 - La modélisation du débat en espace de contraintes

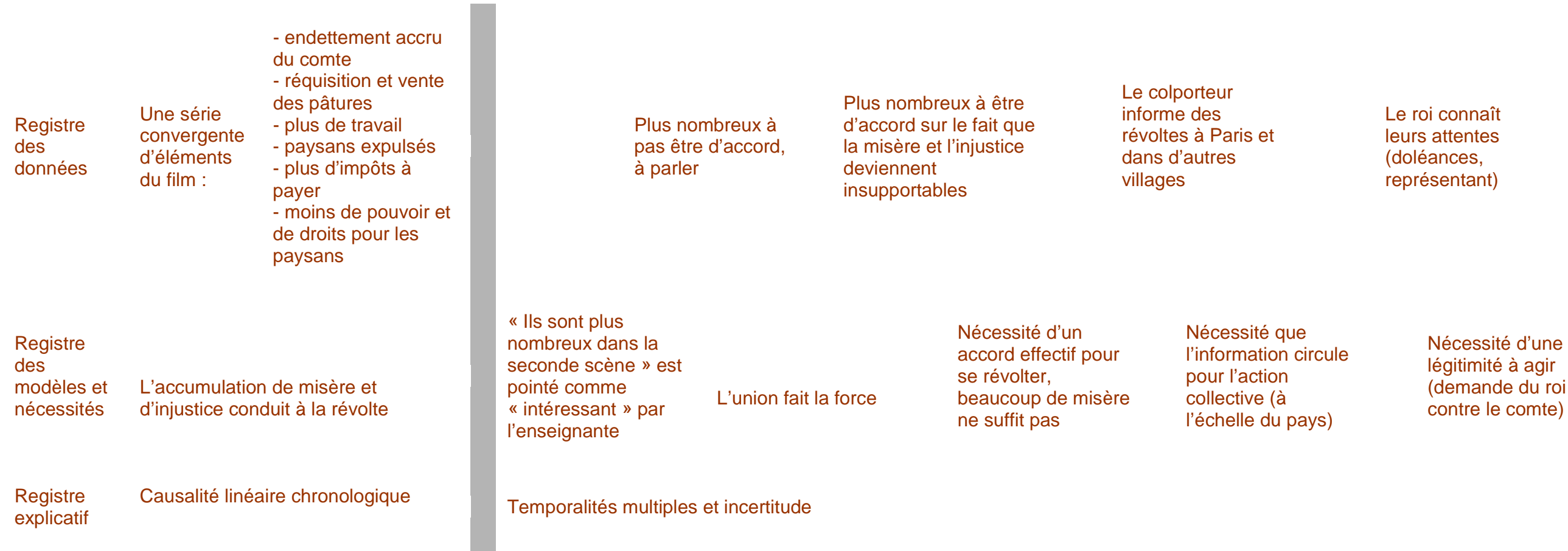


On peut débuter l'analyse de ce cas en observant l'évolution du registre explicatif au cours de la discussion (étudiée à partir de sa transcription, dont un large extrait est donné en annexe). La mise en tension des données et des modèles qui caractérise le processus de problématisation n'intervient que dans la seconde partie du débat. Auparavant (séparation temporelle marquée par la colonne grise), les données et les modèles sont mis en relation sans tension : les élèves cherchent dans le film les données qui permettent de confirmer le modèle explicatif déjà-là (la misère et l'injustice conduisent à la révolte) dans une relation causale linéaire, selon laquelle les comportements sont déterminés par la rencontre mécanique entre un contexte et des individus porteurs de modèles de comportement).

On discerne ici que cette activité de mise en relation va de soi pour les élèves, malgré les signes produits par l'enseignante pour indiquer aux élèves que ces réponses ne sont pas satisfaisantes. Elle intervient en effet régulièrement dans ce sens dans la première partie du débat ${ }^{1}$ :

- « Ça veut dire que non seulement il y a plus d'impôts dans l'histoire de ce village-là, il y a plus d'impôts mais en plus ils ont moins de pâturages. Et qu'est-ce qu'ils disent par rapport à ça ? Ils disent que... que... il y a une expression qui revient souvent par rapport à ça, qui montre que c'est nouveau... Je vous la donne : ils disent « de tout temps »; « de tout temps, nos parents nos ancêtres, menaient les bêtes dans ce pré ». Je ne sais pas si ça vous dit quelque chose ? » (tour de parole 33);

- «Maintenant j'ai juste une question. Est-ce qu'on peut essayer de justifier : pourquoi est-ce que le fait de payer plus d'impôts nous permet de comprendre pourquoi ils se révoltent ? En fait l'idée c'est ça c'est d'essayer d'aller au bout de l'explication » (tour de parole 51) ;

- « lls en ont marre. D'accord. Donc là l'idée c'est d'essayer de comprendre pourquoi ils en ont marre en 1789 et pas en 1788. Est-ce qu'en 1788 ils en avaient déjà marre ou pas ? » (tour de parole 59).

Bien que le dispositif de la séance vise à poser un problème - l'insuffisance de l'explication par la misère et l'injustice - en confrontant les deux scènes du film, l'inertie de la discipline scolaire déjà endossée par ces élèves de fin de primaire, reprend immédiatement ses droits. Le registre explicatif de la linéarité causale qui rend compte de cette inertie leur fournit les ressources pour élaborer des réponses en ajoutant à la succession chronologique la durée comme productrice de phénomènes. Ils expliquent le changement entre 1788 et 1789 par l'accumulation de colère due à l'accroissement de la misère et de l'injustice : le modèle de la " goutte d'eau qui fait déborder le vase » (comme formulé par des élèves dans une situation similaire ${ }^{2}$ ) est disponible pour conforter celui de la misère et de l'injustice et éviter toute tension avec les données sélectionnées dans le film. À ce stade de la séance, le problème est posé par le professeur, mais n'est pas approprié par la classe : données et idées explicatives ne se contrôlent pas les unes les autres mais se renforcent.

Ces constats menés sur la première partie de la discussion collective permettent de nuancer l'écart pointé dans les travaux didactiques sur l'apprentissage de la pensée historienne, entre les capacités des élèves et le travail historien souvent réduit à un processus de contextualisation ${ }^{3}$. Ici les élèves opèrent une certaine mise en contexte des actions discutées : ils les situent entre elles, dans les rapports sociaux qu'ils connaissent de cette période (tels que la soumission des paysans au conte que confirment un certain nombre de scènes du film), et dans un environnement de précarité et de solidarité du monde rural de l'Ancien Régime. Plus encore, la modélisation en terme de problématisation rend visible le fait que l'enjeu de contextualisation du travail historique porte sur le modèle explicatif plutôt que sur l'événement (la révolte). En effet, s'il est difficile d'imaginer que des élèves appréhendent des actions sans contexte (ce que peut laisser supposer l'idée de contextualisation des événements), ils inscrivent en revanche un modèle explicatif dans un contexte ad hoc, non questionné et tout fait, en sélectionnant dans les données celles qui ne le contredisent pas. Autrement dit, dans notre modélisation, les données

\footnotetext{
${ }^{1}$ En annexe est présentée l'essentiel de la transcription des échanges de cette troisième séance.

2 Voir Doussot (2011, p. 125).

${ }^{3}$ Voir notamment sur ce point les travaux fondateurs de Wineburg (1991) qui fait de la contextualisation une des trois « heuristiques » propre au travail historien des sources, Hassani Idrissi (2005) ou encore Martineau (1999).
} 
d'un problème historique concernent aussi bien l'action que le contexte, ce qui tend à réduire l'intérêt analytique de cette dichotomie - issue d'une modélisation non reconnue comme telle au profit de la différenciation explicite entre données et modèle: un problème historique se déploie lorsqu'un modèle est historicisé par confrontation à des données qui en récuse l'évidence explicative.

C'est ce qui se produit dans la seconde partie de la discussion collective, lorsque registre des données et registre des modèles entrent en tension et produisent des nécessités. On le discerne dans partie droite de l'espace de contraintes (tableau 1) par le fait que les élèves font appel à des données du film précédemment ignorées (lors des deux premières séances et lors du début de la discussion), comme le rôle du colporteur, les révoltes ailleurs en France que ce dernier évoque et qui ne sont pas visibles dans le film, mais également à des modèles de comportement nouveaux, les uns et les autres se renforçant mutuellement. Trois conditions à l'usage du modèle explicatif émergent, que l'on peut considérer comme le développement du savoir sur l'événement : sachant que les paysans sont dans la misère et l'injustice depuis très longtemps, il faut, pour qu'ils se révoltent, qu'un accord collectif intervienne sur cette situation de souffrance, qu'une circulation de l'information se produise, et qu'ils ressentent une légitimité à leur action.

La mise en tension est visible lorsqu'on revient à la transcription des échanges. Il y a un basculement, à un moment donné, lorsque l'enseignante souligne (tdp 63) qu'une proposition d'élève est, enfin, intéressante ; la seconde scène, à l'été 1789 , montrant que les paysans en colère sont plus nombreux que dans la première :

- Ben ils sont plus nombreux.

- Tu dis ils sont plus nombreux. Ça c'est intéressant. On va noter ça, qu'est-ce que tu veux dire par là ?

- Les tours de paroles qui suivent concentrent alors cet appel à de nouvelles données et à de nouveaux modèles, et la production des nécessités. On discerne à partir de là un enchaînement entre données et modèles de comportement (tours de parole d'élèves, entre 66 et 88) :

- Ben ils sont plus nombreux à être pas d'accord.

- Comme ils sont nombreux eh bien ils pensent qu'ils ont plus de chances

- Ils sont plus nombreux... ils sont toujours autant mais ils sont... heu il y en a avant qui disaient rien parce qu'ils avaient peur de se faire... enfin

- Ils sont plus nombreux à être d'accord sur le fait qu'ils en ont marre. Et que du coup heu.

- Et après, avant ils étaient concentrés sur leur travail mais maintenant vu que il y a beaucoup plus de monde qui a compris, vu qu'ils se sont fait chasser et tout, ça a accéléré le mouvement.

- Au début ils n'avaient pas tous le même problème et puis après ils étaient plus nombreux à avoir le même problème.

- En fait ils sont plus nombreux c'est... il y a plus de villages qui sont d'accord sur le fait de se révolter.

L'explication qui, dans la première partie, joint l'accumulation de la colère et le modèle qui l'interprète (la goutte d'eau qui fait déborder le vase) devient dans cette deuxième partie une donnée: le fait que la colère s'accumule ne devient explicatif qu'à condition que cette accumulation soit vécue collectivement, avec de multiples conséquences (espoir, courage...). Dans un tel processus, le déterminisme des modèles de comportement est récusé par l'exploration de leurs conditions de possibilité (à quelles conditions le fait d'être plus nombreux a un effet sur la révolte de 1789), qui passe elle-même par un retour aux données. La remémoration du film indique alors non seulement le nombre des révoltés, mais leur accord sur les raisons de la révolte (scènes de discussion entre paysans) et la connaissance qu'ils ont de révoltes au même moment ailleurs (le rôle du colporteur est alors invoqué); ces données prennent sens comme indices en appui sur de nouveaux modèles de comportements (l'union fait la force, la circulation de l'information est essentielle). Autrement dit, dans cette phase du travail de la classe, en «avoir marre » ne suffit plus à déclencher, mécaniquement, la révolte, il faut 
qu'un accord se fasse sur le niveau de colère, ce qui est visible, pour les élèves, dans les échanges entre les personnages du film.

Cette mise en tension ouvre donc, sur le plan historiographique, à une interprétation renouvelée et d'une nature apodictique, selon laquelle la Révolution s'explique en partie par le processus même de la réunion des états généraux déclenchée par le pouvoir royal. L'explication n'est plus déterministe, causale et linéaire, mais articule différentes temporalités (la communication de l'information, les événements extérieurs au village - prise de la Bastille, révoltes contre les seigneurs dans d'autres villages - les actions individuelles et collectives des paysans qui réinterprètent les événements antérieurs comme la rédaction des cahiers de doléances et l'envoi d'un représentant à Versailles), dans un registre explicatif qui donne à voir le champ des possibles de ces hommes, de ce qu'ils peuvent faire, dire, mais aussi de l'interprétation qu'ils peuvent faire eux-mêmes de leur situation. Ainsi, la révolte devient une possibilité dans l'esprit des paysans de ce village lorsqu'ils apprennent que d'autres se révoltent contre leur seigneur, lorsque quelques-uns d'entre eux posent des premiers gestes de révolte, mais également lorsque l'espoir né de la rédaction des cahiers de doléances et de la réunion des états généraux est déçu ; autant d'événements qui ont leur temporalité propre et qui s'influencent plus ou moins directement.

\section{Différenciation données/modèles et habitudes disciplinaires}

Comment expliquer le basculement, au cours de la discussion, qui conduit à une telle mise en tension entre données et modèles explicatifs ? La modélisation interprétative représentée par l'espace de contraintes permet de mettre en évidence le moment de la situation au cours duquel se déclenchent les mises en tension qui marquent le processus de reconstruction du problème de la classe. Schématiquement, on discerne dans ces échanges (tdp 62 à 106) :

- L'énonciation par un élève d'une réponse correspondant à la tâche scolaire proposée, qui s'inscrit dans le cadre ordinaire de la discipline : un jeu de questions/réponses. Ici, la question est celle de la différence entre les deux scènes, la réponse de l'élève est «ils sont plus nombreux » (dans la deuxième scène, en 1789).

- La reprise de cet énoncé qui est non pas validé comme vrai par l'enseignante (selon le modèle dominant de la boucle didactique ${ }^{4}$ ) mais comme «intéressant ». II passe ainsi du statut de réponse à une question, à celui de donnée pertinente dans le problème que l'enseignante tente de poser dans la classe depuis le début de la séance (pourquoi ne se sont-ils pas révoltés dès 1788 , sachant qu'il y avait déjà de la misère et de l'injustice ?).

- La sélection par l'enseignante de l'énoncé « ils sont plus nombreux » focalise l'attention des élèves à une échelle plus locale pour penser l'événement : non pas la révolte qui s'étale sur l'ensemble du film (c'est-à-dire sur une année de vie des personnages), mais ce moment de la révolte qui rassemble l'ensemble du village; non pas la mise à sac du château, mais le regroupement des paysans qui le rend possible.

- À cette échelle, le modèle explicatif dominant n'est plus performant pour interpréter cette donnée «intéressante »: ce ne sont pas la misère et l'injustice qui expliquent le regroupement de tout le village à ce moment-là. Cette tension entre les deux conduit à la recherche d'autres modèles explicatifs. D'où les tentatives des élèves : être plus nombreux donne «plus de chance » de gagner $(72,74)$, enlève «la peur » $(78,102)$, donne « du courage » (100), et s'explique parce qu'ils savent que d'autres villages et Paris se révoltent aussi (88).

- À ce stade des échanges, après la première partie du débat qui explicitait le fait qu'il y avait plus de misère et d'injustice en 1789 qu'en 1788, ces énoncés ancrés dans de nouveaux

\footnotetext{
${ }^{4}$ Ce modèle de la didactique de l'histoire « (question/réponse/ évaluation/formalisation/compléments) donne à chaque protagoniste une place spécifique : l'enseignant contrôle de fait l'argumentation didactique" en disant le "vrai" et assoit, par son propos, l'autorité" des savoirs comme la sienne ; l'élève, le plus souvent privé de réelle prise en charge énonciative, est invité à I'“adhésion" d'un "texte" à apprendre » (Lautier \& Allieu-Mary, 2008, p. 112).
} 
modèles explicatifs ont le statut de conditions de possibilité de l'explication initiale : ils disent que la misère et l'injustice sont des explications de la révolte à condition que les paysans se concertent et comprennent qu'ils sont nombreux à ne pas être d'accord, et qu'ils ont plus de chances de gagner.

- L'autorité de l'enseignante et le fonctionnement habituel de la classe d'histoire (aller chercher des informations dans les documents) conduisent donc à la production d'énoncés différenciés en données et modèles explicatifs : à partir de la donnée initiale (« plus nombreux »), vers une série de modèles explicatifs, mais aussi ponctuellement vers la recherche d'autres données prises dans le film et ignorées jusque-là (par exemple au tdp 91-92 : M : comment ça se fait que tu dis ça et que dans le film ils peuvent savoir ça? El. : Ben parce que il y a des messagers... le colporteur).

Il apparaît que l'insistance sur la pertinence d'une donnée détachée de toute interprétation - ils sont plus nombreux, on peut les compter dans chacune des deux scènes - favorise la recherche d'autres données et d'autres modèles explicatifs. Mais si la modélisation en terme de problématisation structure cette analyse, elle n'est cependant pas seule en jeu dans l'analyse. Les concepts qui la constituent s'articulent constamment à une temporalité qui déborde celle de cette séquence, la temporalité du fonctionnement habituel de la classe d'histoire ${ }^{5}$. Ainsi le processus de différenciation mis au jour s'appuie-t-il directement sur l'habitude qu'ont les élèves de répondre à des questions, en particulier de chercher de l'information dans un document (ici dans le film) et de sélectionner dans leur connaissance du monde social des explications habituelles (fondées sur le sens commun) ${ }^{6}$. Une telle intrication entre les deux temporalités dans lesquelles s'inscrivent les activités des élèves et de l'enseignante conduit à reconsidérer la modélisation elle-même, du moins en ce qui concerne le registre explicatif et son évolution. En effet, si les habitudes disciplinaires s'allient au dispositif singulier (construit en vue d'une problématisation scolaire) pour rendre possible la différenciation inhabituelle qui s'opère entre données et modèles, en revanche, il est douteux que la situation forcée ait permis la remise en cause du registre explicatif chrono-causal habituel des élèves. On le constate en observant les énoncés qui résultent de la différenciation opérée : ils relèvent du raisonnement post hoc qui correspond au type d'argumentation que la classe d'histoire favorise habituellement ${ }^{7}$. Et l'explication initiale fondée sur la misère et l'injustice co-existe certes avec l'explication nouvelle fondée sur la conjoncture politique de l'année 1789 , ce qui relève d'un registre explicatif de temporalités multiples, mais cette co-existence n'est que virtuelle et formulée dans l'analyse didactique : les élèves n'ont pas, quant à eux, l'occasion de confronter leur explication initiale aux propositions qui émergent dans le moment charnière de la discussion. Les multiples explications causales qui ont cours dans la classe ne sont pas l'objet d'un travail explicite qui permettrait de faire de l'articulation des deux temporalités un enjeu; il est probable qu'à leurs yeux une explication se substitue à l'autre comme dans le cours habituel de la classe d'histoire.

\section{Le temps de la différenciation et le temps de l'exploration/cartographie des possibles}

On peut tenter d'interpréter ces constats en revenant à la modélisation des savoirs que constitue la problématisation, au-delà de la seule différenciation/mise en tension des données et des modèles explicatifs, en mettant à nouveau en jeu l'idée d'exploration et de cartographie des possibles. La différenciation soutenue par la focalisation de l'enseignante sur une donnée de la situation permet une exploration de nouveaux possibles au-delà de l'explication initiale, mais le dispositif de la discussion orale en classe entière ne permet pas un travail systématique sur leurs relations, parce qu'il n'y a pas de retour explicite et guidé de comparaison des explications possibles. Dès lors, les élèves n'ont pas l'occasion de confronter ces possibles ni de questionner

\footnotetext{
${ }^{5}$ Et qui renvoie à la notion de discipline scolaire et à sa prégnance dans les pratiques (Tutiaux-Guillon, 2008).

${ }^{6}$ Ce sont les deux opérations qui dominent largement les pratiques ordinaires de la classe d'histoire (Tutiaux-Guillon, 2009).

${ }^{7}$ Par exemple, la connaissance d'autres révoltes précède et explique l'accroissement du courage, le fait de se regrouper induit l'augmentation des chances de succès de la révolte, et l'espoir suscité par les états généraux mène à la déception.
} 
les manières dont ils les articulent habituellement (en faisant jouer la chronologie et la durée, comme nous l'avons évoqué précédemment). On peut dire que le travail de différenciation se produit à une temporalité plus courte que celle qui serait nécessaire à l'exploration et la cartographie des possibles. II semble donc que si l'on vise à considérer le processus de problématisation dans toutes ses dimensions, on a intérêt à penser la modélisation en relation avec l'histoire de la classe, c'est-à-dire en prenant en compte l'historicité dans laquelle se déploie le processus d'enseignement-apprentissage.

À une échelle temporelle réduite, celle de la différenciation entre données et modèles explicatifs dans la troisième séance, nous l'avons détaillé, le dispositif prend le temps de déployer une explication explicite - l'accroissement de la misère et de l'injustice - qui est mise en cause par la focalisation sur un moment de la révolte. Le second mouvement de la discussion collective prend sens en relation avec le premier : ce qui est « intéressant » dans la description du nombre, c'est qu'elle dit autre chose que l'accroissement de la misère et de l'injustice pour comparer 1788 et 1789 dans ce village. On peut dire que la scène qui montre le regroupement de tout le village fait cas pour les élèves dans la mesure où elle constitue une donnée indéniable de la situation (en singularisant 1789 par rapport à 1788) qui n'est pas réductible au modèle explicatif dominant : il devient nécessaire d'expliquer cette différence factuelle. On peut donc dire que la mise en tension consiste ici en la production d'un cas, par contraste avec la manière habituelle de sélectionner des données dans des documents pour en faire des illustrations des modèles explicatifs dominants.

Mais à une échelle temporelle plus large, celle de la production d'une explication issue de l'exploration des possibles, la configuration reste celle de l'illustration. Le dispositif ne prévoit pas de retour explicite des élèves sur l'explication initiale telle qu'elle est produite par le travail des deux premières séances sur le film. La modalité habituelle de production d'explication, ancrée dans le registre chrono-causal, n'est donc pas mise en jeu dans l'activité de la classe alors même que des éléments de sa mise en cause potentielle sont présents et identifiés par l'analyse didactique.

Pour aller plus loin dans l'interprétation de cet écart, il nous semble nécessaire d'étendre la réflexion, à nouveau, à la comparaison entre problématisation scolaire et problématisation scientifique, en retournant à cette dernière, armés du questionnement sur l'historicité des processus de problématisation. Comment, dans les communautés scientifiques, ces différents niveaux de temporalité sont-ils pris en charge?

\section{Différenciation des données et des modèles, registres explicatifs et paradigmes}

Parmi les énoncés qui circulent dans le travail de recherche historique, certains concernent les relations entre les idées explicatives et les données empiriques, tandis que d'autres énoncés concernent les relations entre les explications possibles qui émanent de ce premier niveau et qui sont ceux qui établissent leurs conditions de possibilité. À ce second niveau, on peut transposer la dichotomie des données et des idées explicatives pour envisager leur mise en tension comme problématisation des manières habituelles d'articuler les explications en jeu : les données sont les explications possibles produites par le travail sur les sources, tandis que les idées portent sur les relations possibles entre explications - c'est-à-dire les registres explicatifs - qui ne dépendent pas seulement de l'objet étudié, mais avant tout de la tradition qui prévaut dans la communauté dans et pour laquelle ce travail est produit.

Cette perspective, en insérant une dimension institutionnelle à l'analyse épistémologique, fait écho à la caractérisation des communautés scientifiques comme institutions de production de textes de savoir dont « l'une des caractéristiques (...) est l'ampleur (relative) des communications à l'intérieur du groupe et son unanimité (relative) de jugement sur les matières 
professionnelles ${ }^{8} \gg($ Kuhn, 1990, p. 394). Il existe des revues, des laboratoires, des séminaires et des collaborations multiples qui organisent une circulation spécifique des textes selon des procédures que l'étude socio-historique des sciences ${ }^{9}$ met au jour dans leur genèse et leur fonctionnement, et qui montrent le rôle essentiel des instruments et des instances de production critique. Ces circulations de textes sont rendues possibles par une connaissance partagée dans la communauté scientifique de la mémoire disciplinaire, de ses corpus de textes et de ses outils habituels - conceptuels ou autres - pour en permettre une évaluation selon les modalités habituelles - paradigmatiques - de construction et de résolution de problèmes dans la discipline. Comme le dit Fleck (2008, p. XXX), les sciences « sont des phénomènes culturels complexes, autrefois peut-être individuels, de nos jours collectif [...]. Une structure organisée spécifique avec ses hiérarchies, ses modalités de communication et de coopération, ses tribunaux internes, son opinion publique et ses instances de décision ».

Un processus de problématisation scientifique peut donc être vu comme la conjonction de deux perspectives temporellement différenciées. L'une, cognitive, rend compte des relations entre les trois registres (empirique, des modèles et explicatif), l'autre, institutionnelle, inscrit les circulations d'énoncés entre les protagonistes de la communauté dans ces différents registres par le biais de la définition des rôles et des fonctions dans cette institution communauté scientifique. Cette conjonction de structures sociales et de structures cognitives explique que « la science est une construction qui fait émerger une découverte irréductible à la construction et aux conditions sociales qui l'ont rendue possible » (Bourdieu, 2001). L'organisation institutionnelle de la circulation des textes dans une discipline constitue celle-ci en communauté scientifique ${ }^{10}$ qui, en se développant, développe un paradigme ${ }^{11}$, qui régule les échanges, qui encadre ce qui va de soi, qui différencie ce qui peut être en question et ce qui doit être hors de question. Dans cette " économie des biens symboliques » scientifiques, les textes de savoir se différencient, parce qu'ils sont dialogiques et argumentatifs, ce que marquent les références aux noms propres, et ce qui repose sur la nécessité institutionnelle de référer aux autres textes ${ }^{12}$. Mais ils sont également le fruit d'un processus d'échange qui s'inscrit dans le temps (que récuse l'image d'immédiateté et d'individualité de la découverte), dans une linéarité temporelle (que marque l'indication de l'année pour référer à un texte) qui n'octroie le statut de découverte qu'en relation à toute la mémoire de la discipline, c'est-à-dire à l'histoire des problématiques du champ. On peut dire qu'un champ scientifique gère la mémoire du champ à travers l'usage - au double sens du terme : la pratique et l'habitude - des concepts de la discipline. En ce sens, la construction de problèmes scientifiques est cadrée par des registres explicatifs disciplinaires appropriés par les membres de la communauté en question, qui sont simultanément les générateurs de la dynamique de mise en tension des données et des idées explicatives qui prennent forme lors des échanges de textes (à de multiples niveaux : individuel par le dialogisme, en petits groupes en séminaires, vers la publicisation par les revues et colloques, etc.). Qu'en est-il alors dans le cas scolaire de la séquence sur la Révolution? Dans quelle mesure l'histoire de la classe est-elle institutionnalisée, gérée et/ou remise en question? Qu'en est-il plus spécifiquement pour l'histoire ${ }^{13}$ ?

\footnotetext{
${ }^{8}$ Kuhn précise que pour distinguer des groupes de travail scientifique marqués par un même paradigme il faut « fréquenter les écoles d'été et les conférences spécialisées, connaître les listes de ceux qui reçoivent les preprints [désigne les article envoyés à des collègues inscrits sur une mailing liste, sous forme ronéotée ou photocopiée, au moment de leur soumission à une revue pour publication (N.d.T.)] et, avant tout, connaître les réseaux d'information formels comme informels, ainsi que les relations entre les diverses citations » (Kuhn, 1990, p. 395).

${ }^{9}$ En histoire des sciences, voir par exemple Shapin (2014).

${ }^{10}$ Qui ne suppose donc pas, dans cette acception, une unité ni une unanimité, mais renvoie au lieu et à l'échelon de travail effectif de recherche sur la base de modalités pratiques et épistémologiques partagées.

${ }^{11}$ Au sens de la problématique de Kuhn $(1983 ; 1990)$ : quelque chose qui guide la validation sans pour autant se réduire à des règles à appliquer, et qu'il identifie finalement à des exemples exemplaires (exemplars) de production de problèmes.

12 «Un des principes de la spécificité du champ scientifique résidant dans le fait que les concurrents ne peuvent pas se contenter de se distinguer de leurs devanciers déjà reconnus, mais sont contraints sous peine d'être dépassés et « déclassés », d'intégrer leurs acquis dans la construction distincte et distinctive qui les dépasse » (Bourdieu, 1975).

${ }^{13}$ Sachant que les travaux de Kuhn portent sur le champ de la physique.
} 


\section{La problématisation historique comme double étude de cas}

Ces caractéristiques épistémologiques et institutionnelles constituent un arrière-plan qui peut nous aider à mobiliser autrement la modélisation du savoir en terme de problématisation sur la séquence étudiée. Le format de la séquence forcée mise en œuvre permet visiblement la gestion de l'histoire locale de la classe - à l'échelle de cette séquence, celle qui se centre sur la production de plusieurs interprétations de l'événement par différenciation entre données et modèles. En revanche, il ne rend pas possible une mise en question des manières de gérer cette multiplicité en laissant jouer le registre explicatif habituel qui associe causalité, chronologie et durée.

En se référant à la problématique de Kuhn et aux propositions historiographiques et épistémologiques de Ginzburg (2010), et en particulier à une de ses principales études historiques (Ginzburg, 1980), on peut éclairer cette analyse par le concept d'étude de cas. Dans son texte qui fit date, Ginzburg (1980) propose en effet ce qu'il appellera par la suite une étude de cas, qui fonctionne à deux niveaux. À un premier niveau, le dossier d'archives du procès en inquisition d'un meunier du Frioul à la fin du XVle siècle met en question le modèle explicatif dominant chez les historiens des années 1970 du rapport entre culture populaire et culture de l'élite. Ce meunier, tel qu'il se montre dans les archives, ne se révèle pas dépendant culturellement des élites notamment religieuses et manifeste une indépendance culturelle liée à des références techniques et matérielles. Ce dossier fait cas, en premier lieu, par rapport à ce qui avait été étudié jusque-là, bien qu'il soit unique : il oblige les historiens à questionner leurs interprétations dominantes. Cependant, la validité de ce cas n'est pas évidente et dépend d'un second niveau d'analyse. Dans le paradigme quantitativiste dominant à l'époque, un seul dossier d'archive n'est pas légitime à mettre en question le savoir tiré de l'analyse sérielle et systématique de quantité de données; il est même a priori considéré comme non représentatif. S'il veut convaincre, Ginzburg est donc dans l'obligation de mettre simultanément en question le paradigme lui-même. Or, comme nous venons de le voir, un paradigme est toujours à la fois ancré épistémologiquement et institutionnellement. La remise en question à ce second niveau est donc nettement plus longue et difficile que la première sur l'objet même de l'enquête (les liens entre culture populaire et culture de l'élite dans l'Italie du Nord à la fin du XVle siècle). La tâche pour l'historien consiste, sur ce plan, à remettre en discussion les modalités habituelles de jugement des manières d'explorer et cartographier les explications possibles (ici, une simple source peut remettre en cause une explication fondée sur une série de sources). Revenons alors à notre séquence.

Si la situation de ce village fait cas dans le dispositif organisé à la troisième séance, c'est parce que les deux premières ont permis de mettre en évidence une explication dominante acceptable et acceptée par les élèves. Dans cette première phase, le film sur ce village constitue une source d'exemples de l'effet de la misère et de l'injustice sur les peuples. Puis l'analyse serrée et micro de deux moments du film permet de le transformer en source de questionnement par le mécanisme de la différenciation entre données et modèles, comme nous l'avons vu. Comme dans le cas du meunier pour l'historien, l'action de regroupement massif de ces villageois oblige les élèves à remettre en discussion l'explication dominante. Mais là s'arrête la comparaison. La courte durée et le fonctionnement oral et collectif immédiat du dispositif de classe ne permettent pas le déploiement d'une remise en cause du type d'explication chrono-causale. Cette comparaison donne toutefois à voir la dimension productive de la modélisation. Elle rend possible la caractérisation de l'enquête historienne comme pratique inscrite dans un temps, un lieu et des pratiques instrumentées variables, afin d'en identifier les principes et de les transposer à la mesure de la classe. Elle ouvre donc à la possibilité de poursuivre l'enquête didactique : de produire de nouvelles situations forcées dans lesquelles certains de ces principes pourront être insérés afin de produire des données nouvelles de compréhension des conditions de possibilité d'une histoire scolaire problématisée. En particulier, elle ouvre à la possibilité de mettre en jeu, explicitement, le registre explicatif chrono-causal de l'histoire scolaire. 


\section{Références}

AUDIGIER François (1995), « Des savoirs scolaires en question entre les définitions officielles et les constructions des élèves », Spirale, n¹5, p.61-89.

BOURDIEU Pierre (1975), « La spécificité du champ scientifique et les conditions sociales du progrès de la raison », Sociologie et sociétés, vol. 7, n¹, p.91-118.

BOURDIEU Pierre (2001), Science de la science et réflexivité, Paris, Raisons d'agir.

CHARTIER Roger (2000), Les origines culturelles de la Révolution française, Paris, Seuil.

DELUERMOZ Quentin \& SINGARAVÉLOU Pierre (2016), Pour une histoire des possibles analyses contrefactuelles et futurs non advenus, Paris, Éditions du Seuil.

DOUSSOT Sylvain (2011), Didactique de l'histoire : outils et pratiques de l'enquête historienne en classe, Rennes, Presses universitaires de Rennes.

DOUSSOT Sylvain (2015), «Film de fiction en classe d'histoire et inégalité des compétences d'interprétation », Spirale, $\mathrm{n}^{\circ} 55$, p.165-177.

FABRE Michel (2009), Philosophie et pédagogie du problème, Paris, Vrin.

FAILEVIC Maurice \& LA ROCHEFOUCAULD (de) Jean-Dominique (1978), 1788 : luttes révolutionnaires pour une propriété paysanne, Paris, Éditions sociales.

FLECK Ludwik (2008), Genèse et développement d'un fait scientifique, Paris, Flammarion.

GINZBURG Carlo (1980), Le fromage et les vers. L'univers d'un meunier du XVI siècle, Paris, Flammarion.

GINZBURG Carlo (2010), Mythes emblèmes traces morphologie et histoire, Paris, Verdier.

HASSANI Idrissi Mohamed (2005), Pensée historienne et apprentissage de l'histoire, Paris, L'Harmattan.

KUHN Thomas S. (1983), La structure des révolutions scientifiques, Paris, Flammarion.

KUHN Thomas S. (1990), La Tension essentielle tradition et changement dans les sciences, Paris, Gallimard.

LAUTIER Nicole (1997), A la rencontre de l'histoire, Villeneuve-d'Ascq, Presses universitaires du Septentrion.

LAUTIER Nicole \& ALLIEU-MARY Nicole (2008), « La didactique de I'histoire », Revue française de pédagogie, $\mathrm{n}^{\circ} 162$, p.95-131.

MARTINEAU Robert (1999), L'histoire à l'école. Matière à penser..., Paris, L'Harmattan.

ORANGE Christian (1997), Problèmes et modélisation en biologie. Quels apprentissages pour le lycée ?, Paris, PUF.

ORANGE Christian (2005), « Problématisation et conceptualisation dans les sciences et dans les apprentissages scientifiques », Les sciences de l'éducation. Pour l'ère nouvelle, vol. 38, n³, p.69-93.

ORANGE Christian (2010), « Situations forcées, recherches didactiques et développement du métier enseignant », Recherches en éducation, $\mathrm{HS} \mathrm{n}^{\circ} 2$, p.73-85.

PROST Antoine (1996), Douze leçons sur l'histoire, Paris, Éditions du Seuil.

REVEL Jacques (2001), «Retour sur l'événement: un itinéraire historiographique », dans Jean-Louis Fabiani (éd.), Le goût de l'enquête : pour Jean-Claude Passeron, Paris, L'harmattan, p.95-118. 
SHAPIN Steven (2014), Une histoire sociale de la vérité science et mondanité dans l'Angleterre du XVII ${ }^{e}$ siècle, Paris, La Découverte.

TUTIAUX-GUILLON Nicole (2008), « Interpréter la stabilité d'une discipline scolaire: I'histoire-géographie dans le secondaire français », dans François Audigier et Nicole Tutiaux-Guillon (éds.), Compétences et contenus. Les curriculum en question, Bruxelles, De Boeck, p.117-146.

TUTIAUX-GUILLON Nicole (2009), «L'histoire scolaire au risque des sociétés en mutation », Raisons Comparaisons Educations. La revue française d'éducation comparée, n4, p.105-118.

WINEBURG Samuel S. (1991), « On the Reading of Historical Texts: Notes on the Breach Between School and Academy », American Educational Research Journal, vol. 28, n³, p 495-519.

\section{Annexe}

Be Parce que en fait déjà à la base ils n'ont pas beaucoup d'argent donc heu... alors leur mettre encore plus d'impôts ça va... ils vont pas pouvoir tous les payer.

Oui c'est comme si c'était un petit peu exagéré. Déjà c'était, déjà la situation était terrible, ce que vous avez dit là : des impôts, écrasés par le travail, la misère, la famine... si en plus on augmente les impôts euh là ça devient trop quoi. Donc qu'est-ce que, qu'est-ce qu'il se passe du point de vue des paysans? Ouais?

el C'est l'injustice

Ça devient totalement injuste, mais ça c'était déjà un peu injuste avant. La situation elle était déjà injuste. Paul tu vas discrètement t'installer ici là, je veux lus t'entendre ; puisque tu ne participes pas tu te mets là et tu te tais. Ouais yaël ?

Ya Du coup ben ils se révoltent.

Et pourquoi alors et du coup ils se révoltent, c'est-à-dire ? Fabiola ?

Fa Ben parce que ils en ont marre de payer.

Ils en ont marre. D'accord. Donc là l'idée c'est d'essayer de comprendre pourquoi ils en ont marre en 1789 et pas en 1788. Est-ce qu'en 1788 ils en avaient déjà marre ou pas ?

els Oui...

Oui ? Mais qu'est-ce qui change ? On essaye d'avancer. Charlotte je te donne la parole puisque tu levais la main.

Ch Ben ils sont plus nombreux.

Tu dis ils sont plus nombreux. Ça c'est intéressant. On va noter ça qu'est-ce que tu veux dire par là ?

Ch Ben ils...

Qu'est-ce que tu veux dire ils sont plus nombreux. Ils sont plus nombreux c'est pas parce qu'il y a eu des paysans qui sont apparus... ils existaient déjà l'année d'avant.

Ch Ben ils sont plus nombreux à être pas d'accord.

Là je pense qu'il y a quelque chose qui peut être vraiment important de comprendre mais... alors on a besoin de tout le monde là. Charlotte a dit quelque chose je pense qu'il faut que tous ensemble on arrive à comprendre ce qu'on peut en dire de ça. Alors Emma. 
Em Ils sont plus nombreux à être d'accord, enfin...

Em oui

... contre. D'accord ? A être on va dire contre heu... contre le comte, etc., contre cette situation, ouais. Charlotte?

Ch Comme ils sont nombreux et bien ils pensent qu'ils ont plus de chances

Ouais, plus de chance... de ? Arnaud?

Ar Plus de chance d'avoir du pouvoir quoi

Ah donc plus nombreux ça veut dire que ça leur donne un sorte de force quoi. Le nombre fait la force. C'est un peu ça que vous voulez dire?

els ?

De... de se faire entendre quoi, oui c'est ça ils ont plus de chance de se faire entendre, de faire entendre ce qu'ils disent, ce qu'ils réclament. Est-ce qu'on peut avancer là-dedans, il y a, sur cette histoire-là ils sont plus nombreux. Comment ça se fait qu'ils disent qu'ils sont plus nombreux dans le village et c'est pas vrai qu'ils sont plus nombreux. En fait ce qu'elle dit Charlotte c'est pas exact... c'est pas tout à fait heu... assez précis, moi je pense qu'il y a un truc très important dans ce qu'elle dit mais il faut qu'on essaye de le préciser. Dans le village ils sont pas plus nombreux les paysans, c'est pas vrai. Charlotte?

Ch Ils sont plus nombreux... ils sont toujours autant mais ils sont... heu il y en a avant qui disaient rien parce qu'ils avaient peur de se faire... enfin

Ah ! Avant ils avaient peur et maintenant ils ont moins peur. Bon mais alors pourquoi, il y a des mains qui se sont levées. Baptiste et ensuite Eliette et Adèle.

el Loup.

Loup, oh, excusez-moi.

Lo Ils sont plus nombreux à être d'accord sur le fait qu'ils en ont marre. Et que du coup heu.

A trouver, oui voilà, plus nombreux à être contre et du coup à être d'accord là-dessus. Alors, Eliette et ensuite Fabiola.

El Et après, avant ils étaient concentrés sur leur travail mais maintenant vu que il y a beaucoup plus de monde qui a compris vu qu'ils se sont fait chassés et tout, ça a accéléré le mouvement.

Alors, faut qu'on avance là-dessus. Je vais donner la parole à Fabiola et puis à Lou et puis on va voir

Fa Au début ils avaient pas tous le même problème et puis après ils étaient plus nombreux à avoir le même problème.

Ah ils étaient plus nombreux. Moi c'est toujours, on est bloqué là-dessus. Dans ce village-là c'est pas vrai, dans ce village là ils se parlaient déjà avant. Ils se connaissent on le voit bien ils se tutoient, ils sont proches, ils sont tous un peu dans la même situation, plus ou moins pauvres, c'est vrai mais. Donc c'est pas vrai qu'ils sont plus nombreux à être vraiment conscient de ça et à en souffrir. Alors, Adèle... Loup!

Lo En fait ils sont plus nombreux c'est... il y a plus de villages qui sont d'accord sur le fait de se révolter.

Là on est en train de prendre un petit peu de recul, on n'est plus sur le village de notre histoire, qui je vous le répète est une histoire inventée, mais on est en train de, d'élargir un peu et de voir quoi alors ? Tu dis d'autres villages... 
Ah il y a des messagers... ah il y a le colporteur qui serait un petit peu messager. Ouais alors qui estce qui veut intervenir maintenant. On avance, ou vas-y Abel

94

Ab Comment il s'appelle le fils là..

Oui, Guillaume

Ab II heu, il les incite à dire heu... il les incite heu... qu'il disent des choses au roi...

Ah, incite à dire des choses au roi, alors là-dessus est-ce qu'on peut être un petit peu plus précis par rapport à ce qui se passe. Alors Baptiste vas-y.

$\mathrm{Ba}$ Ben en fait le colporteur il leur dit que à Paris et ben ils se révoltent, et du coup ça les entraîne à se révolter

Ça les entraîne à se révolter. C'est-à-dire, qu'est-ce que tu veux dire par là ?

Ba Ben en fait ben ça leur donne du courage, heu...

D'accord

el Ils ont moins peur

Ah, ils ont moins peur parce qu'ils savent, grâce au colporteur, qu'il y a eu une révolte à Paris.

$\mathrm{Ba}$ Donc qu'ils sont plus tout seuls

Et donc ils sont plus tout seuls. Plus nombreux, plus tout seuls. Ils savent grâce au colporteur qu'ailleurs en France et même à Paris, il y a des révoltes. Est-ce qu'on peut même carrément être plus précis par rapport à cette histoire de révolte à Paris ?

Lo La prise de la Bastille 